\title{
COMPLICACIONES EN 748 HISTERECTOMÍAS POR LAPAROSCOPIA UTILIZANDO UN MANIPULADOR UTERINO CON RESALTADOR VAGINAL
}

\section{Complications in 748 laparoscopic hysterectomies using an uterine manipulator with vaginal highlighter}

Ramiro Argüello-Argüello, $M D^{I}$

Recibido: noviembre 11/11 - Aceptado: septiembre 16/12

\section{RESUMEN}

Introducción: la histerectomía laparoscópica (HL) requiere utilizar un dispositivo que permite una sección segura de la cúpula vaginal. El autor desarrolló un manipulador uterino con resaltador vaginal (M-R) para este paso. El objetivo de este trabajo es presentar las complicaciones asociadas a la utilización del M-R en diez años de uso.

Materiales y métodos: se presenta una cohorte de mujeres sometidas a histerectomía laparoscópica en quienes se utilizó el manipulador uterino con resaltador vaginal (M-R) entre diciembre 7 de 1999 y junio 21 de 2011. El criterio de inclusión fue indicación de histerectomía por patología benigna. Se excluyeron aquellas con indicación de histerectomía vaginal, sospecha de adherencias intraabdominales o úteros excesivamente grandes. Se evaluó la edad, la paridad, el antecedente de cesáreas o laparotomías previas, el tiempo quirúrgico, el peso de la pieza quirúrgica, las complicaciones intra o posoperatorias y la evolución durante un seguimiento mínimo de 30 días posteriores a la cirugía.
Resultados: en el periodo de observación se operaron 854 pacientes. En 106 pacientes el seguimiento posoperatorio fue menor a un mes. Los datos presentados corresponden a 748 pacientes. La edad promedio de las pacientes fue de 44 años $( \pm 6,6)$. Las principales indicaciones de HL fueron miomatosis y adenomiosis uterina. Tiempo quirúrgico promedio: $90 \mathrm{~min}$ ( \pm 17,7). Peso uterino promedio: $205 \mathrm{~g}( \pm 88,3)$. Se presentaron complicaciones en 41 pacientes $(5,6 \%)$, de las cuales seis $(0,8 \%)$ fueron complicaciones mayores: tres lesiones vesicales $(0,4 \%)$, una lesión ureteral $(0,13 \%)$, una paciente $(0,13 \%)$ requirió laparoscopia operatoria por sangrado posquirúrgico, y una paciente $(0,13 \%)$ laparotomía exploratoria por dolor abdominal. La tasa de conversión a laparotomía fue de 2\%.

Conclusión: con el uso del M-R se presentaron tasas de complicaciones similares a las reportadas por otros autores.

Palabras clave: histerectomía por laparoscopia, histerectomía total por laparoscopia, manipulador uterino, complicaciones.

Médico ginecólogo. Experto en Endoscopia Ginecológica. Director Científico de Ginelap. ramiroarguellomd@gmail.com 


\section{ABSTRACT}

Introduction: Laparoscopic hysterectomy (LH) involves using a device which permits safe section of the vaginal dome. The present author has developed an uterine manipulator with vaginal highlighter (UM-VH) for this step. The present work has been aimed at presenting complications associated with using the UM-VH which have arisen during 10 years' use.

Materials and methods: This study involved a cohort of women undergoing laparoscopic hysterectomy using a UM-VH between December $7^{\text {th }} 1999$ and June $21^{\text {st }} 2011$. Inclusion criteria consisted of an indication of hysterectomy due to benign pathology. Females were excluded who had an indication of vaginal hysterectomy or where there was suspicion of excesively large uterine or intraabdominal adherences. Age, parity, a background of prior caesarean sections or laparotomy, time spent in surgery, the weight of the surgically-excised piece and intra- or post-operatory complications were evaluated and evolution was followed-up for at least 30 days after surgery.

Results: 854 patients were operated on during the observation period. Post-operatory follow-up was less than a month for 106 patients; the data presented here deals with the remaining 748 patients. The patients' average age was 44 years $( \pm$ 6.6); uterine myomatosis and adenomyosis were the main indications for LH. Average time spent in surgery was 90 minutes $( \pm 17.7)$. Average uterine weight was 205 grams ( \pm 88.3). Complications occurred in 41 patients (5.6\%), six of which were serious complications (0.8\%): three vesical lesions $(0.4 \%)$, a urethral lesion $(0.13 \%)$, one $(0.13 \%)$ patient required operative laparoscopy due to bleeding after surgery and one (0.13\%) patient underwent exploratory laparotomy because of abdominal pain. There was a $2 \%$ conversion to laparotomy rate.

Conclusion: Using UM-VH has led to similar complication rates to those reported by other authors.
Key words: Laparoscopic hysterectomy, total laparoscopic hysterectomy, uterine manipulator, complicacions.

\section{INTRODUCCIÓN}

La histerectomía es la cirugía mayor ginecológica más frecuentemente realizada en el mundo $(1,2)$. Hoy en día se reconoce que la vía vaginal se debe preferir a la abdominal y que, cuando no es posible la vía vaginal, la laparoscopia es mejor que la vía abierta $(1,3)$. Uno de los factores que han influido para lograr una mayor aceptación de la histerectomía laparoscópica (HL) es el desarrollo de diferentes técnicas quirúrgicas para este tipo de histerectomía (4-6), y la introducción de dispositivos que permiten mejorar la movilización del útero durante la cirugía y la identificación clara de la unión vagino-cervical en el momento de realizar la colpotomía, disminuyendo así la posibilidad de lesión vesico-ureteral y los tiempos quirúrgicos (7-12).

La técnica inicial para realizar la histerectomía por laparoscopia no consideraba, inicialmente, ningún dispositivo especial para resaltar el reborde vagino-cervical. Es por esto que predominó la histerectomía vaginal asistida por laparoscopia $(5,6)$, que corresponde a la Tipo I de Munro (4). En esta técnica quirúrgica la parte laparoscópica se limitaba a la liberación del útero hasta antes de la sección de las arterias uterinas (5). Muchos consideraban esta cirugía laparoscópica como un paso innecesario de una cirugía vaginal (12). Estos inconvenientes empezaron a resolverse una vez se logró visualizar durante la laparoscopia el reborde vaginal en su punto de unión con el cérvix.

La clara identificación de la unión vagino-cervical durante la cirugía laparoscópica es importante como punto de referencia, pues evita disecar excesivamente la vejiga y los tejidos parametriales. Por medio de esta maniobra se logra: a) incrementar la longitud de la vagina 2 a $3 \mathrm{~cm}$ en el momento del corte, b) separar los uréteres del trayecto del corte por la tensión aplicada, y c) realizar la sección de la colpotomía sin seccionar los ligamentos cardinales 
ni los ligamentos útero-sacros, preservando los ligamentos de sostén de la vagina y disminuyendo la posibilidad de daño ureteral en el momento del cierre de la cúpula vaginal (11). Por otra parte, también se requiere conservar el neumoperitoneo en el momento de abrir la cúpula vaginal y poder movilizar adecuadamente el útero en diferentes direcciones con el fin de exponer la zona operatoria. Además, se requiere que los dispositivos utilizados sean fáciles de ensamblar y de usar, y que en lo posible sean de bajo costo.

Se han diseñado dispositivos con el objetivo de lograr estas funciones, por ejemplo, Mueller describe su experiencia con el instrumento de Hohl el cual se atornilla en el cérvix y sobre su eje se fija el adaptador cervical que también sirve como neumo-oclusor (8). Lee describe el uso del modelo de Biswas, el cual se introduce dentro del útero atornillando el eje en el miometrio y colocando el resaltador sobre el eje (9). Keriakos informa el uso de un resaltador que se monta sobre el movilizador de Rumi (13). Corvalán en una publicación, y Perino en otra informan el uso del manipulador uterino de Clermont-Ferrand, el cual utiliza anillos de goma en la porción vaginal con el fin de lograr la neumo-oclusión $(14,15)$. Tanprasertkul describe su experiencia con el manipulador uterino de Anurach, el cual adapta una sonda de Foley No. 26 como neumo-oclusora, montada sobre un eje de acero inoxidable que se fija, junto con el resaltador, mediante una sutura en el cérvix (7).

Mettler y Nikam compararon diferentes movilizadores, entre ellos el de Clermont-Ferrand, el de Rumi-Koh y el de Hohl, y concluyen que aún no se ha diseñado el movilizador uterino que reúna todas las características deseables en este dispositivo (16).

En Colombia, el autor desarrolló el manipulador uterino con resaltador vaginal (M-R) (17). El M-R consta de una varilla de acero inoxidable de $46 \mathrm{~cm}$ de largo y $5 \mathrm{~mm}$ de grosor, con una curva que produce una cavidad cóncava a los $18 \mathrm{~cm}$, y un asa de manipulación en su extremo distal. Sobre la varilla se coloca un tubo de teflón cerrado en su punta que consta de un balón distal que se puede llenar hasta $10 \mathrm{cc}$, y otro balón proximal que permite llenados de 100 a 120 cc. Entre estos dos balones se coloca el resaltador vaginal el cual se construyó con polímeros utilizados en odontología resistentes al calor generado por una unidad electro-quirúrgica, y no conductores de la electricidad. Contiene un reborde periférico a $0,5 \mathrm{~cm}$ del extremo distal que permite una mejor visualización laparoscópica del reborde vagino-cervical (figura 1). El costo promedio es cercano a 24.000 pesos (USD12,5), incluyendo partes desechables y reutilizables.

Figura 1.

Manipulador uterino con resaltador vaginal (M-R)
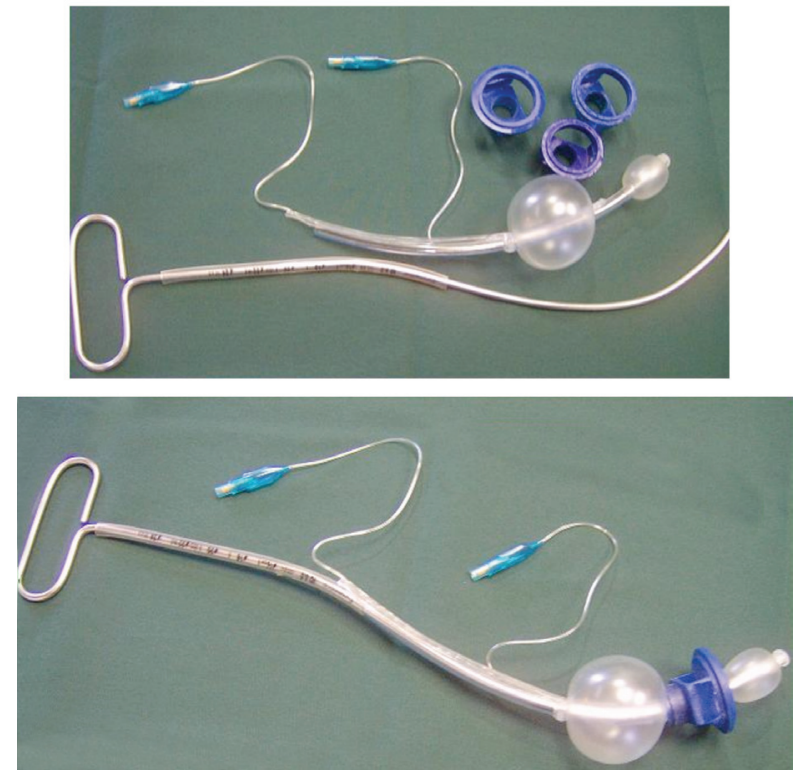

El objetivo de este artículo es presentar los resultados en términos de complicaciones de las pacientes a quienes se les realizó HL utilizando el dispositivo M-R en los últimos diez años.

\section{MATERIALES Y MÉTODOS}

Se presenta una cohorte de mujeres sometidas a histerectomía laparoscópica en quienes se utilizó el manipulador uterino con resaltador vaginal entre el 7 de diciembre de 1999 y el 21 de junio de 2011, enviadas para cirugía a la Unidad de Endoscopia 
Ginecológica. Esta es una institución privada que atiende pacientes de aseguramiento contributivo y privado en Bogotá, Colombia.

Se incluyeron pacientes con indicación de histerectomía por patología uterina benigna. Como criterios de exclusión para HL se consideraron la indicación de histerectomía vaginal, sospecha de adherencias pélvicas severas dadas por útero fijo al tacto vaginal o antecedentes quirúrgicos, y las pacientes con úteros excesivamente grandes en las que en el examen pélvico se consideró extracción difícil o imposible por vía vaginal.

La técnica quirúrgica de la HL ya ha sido descrita (18). Actualmente damos preferencia al cierre de la cúpula vaginal con nudos intracorpóreos laparoscópicos y no por vía vaginal. En todas las cirugías participó el autor.

Para la recolección de los datos se diseñó un formulario especial. Se recolectó la información respecto a la edad, la paridad, los antecedentes de cesáreas o laparotomías previas, el tiempo quirúrgico, el peso de la pieza quirúrgica, las complicaciones intra o posoperatorias, y la evolución durante un seguimiento mínimo de 30 días posteriores a la cirugía. Se clasificaron según los criterios utilizados en el meta-análisis realizado por Chapron (19). Para el análisis de la información se exportó la base de datos a Excel ${ }^{\circledR}$ y a SPSS $19 \circledR$.

Las variables continuas se describieron a través de medidas de tendencia central y dispersión, y la distribución de frecuencias se describió utilizando proporciones.

El estudio fue aprobado por el Comité de Ética de la Unidad, y todas las pacientes aceptaron el uso del dispositivo al firmar el consentimiento informado autorizando la cirugía.

\section{RESULTADOS}

Entre 7 de diciembre de 1999 y el 21 de junio de 2011 se realizaron 854 HL utilizando el M-R. En 106 pacientes $(12,4 \%)$ no se logró recolectar la información a los 30 días de seguimiento. Por tanto, se analizan 748 pacientes.
La edad promedio de las pacientes fue de 44 años $( \pm 6,6)$; el promedio de paridad fue de 2 (rango 0-14); 241 pacientes (32\%) tenían antecedente de cesárea (rango 0-4); 60 pacientes (8\%) tenían laparotomía anterior; la duración promedio de la cirugía fue de $90 \mathrm{~min}( \pm 17,7)$ y el peso promedio uterino fue de $205 \mathrm{~g}$ ( $\pm 88,3)$.

Los diagnósticos prequirúrgicos y los hallazgos de patología se describen en la tabla 1. Las lesiones de alto grado se llevaron a HL con conización previa excluyendo cáncer de cuello, pero a pesar de este requerimiento se encontraron cuatro $(0,5 \%)$ lesiones malignas cervicales. Se encontró un adenocarcinoma cervical no sospechado en una paciente con indicación quirúrgica de miomatosis para la histerectomía. Las pacientes con resultados de patología maligna se remitieron para manejo complementario a unidades de oncología.

\begin{tabular}{|l|c|c|}
\hline \multicolumn{2}{|c|}{ Tabla 1. } \\
Diagnósticos prequirúrgicos y resultados \\
de patoloía
\end{tabular}

En la tabla 2 se presentan las complicaciones encontradas. Se presentaron complicaciones mayores en seis pacientes $(0,8 \%)$, de las cuales tres $(0,4 \%)$ correspondieron a pacientes con lesiones vesicales, una $(0,13 \%)$ con daño ureteral, una $(0,13 \%)$ con sangrado posperatorio que requirió laparoscopia posterior, y una $(0,13 \%)$ paciente con laparotomía exploratoria.

Las complicaciones infecciosas correspondieron a abscesos de cúpula. Las complicaciones hemorrá- 


\begin{tabular}{|l|c|c|}
\hline \multicolumn{3}{|c|}{ Tabla 2. } \\
\multicolumn{3}{|c|}{ Resumen de complicaciones por categoría, } \\
frecuencia y porcentaje \\
\hline \multicolumn{2}{|c|}{ Complicaciones mayores y menores } \\
\hline Complicación & Frecuencia & $\%$ \\
\hline Infecciosas & 15 & 2 \\
\hline Hemorrágicas & 11 & 1,5 \\
\hline Vesicales & 3 & 0,4 \\
\hline Uréter & 1 & 0,1 \\
\hline Otras & 12 & 1,6 \\
\hline Total & 42 & 5,6 \\
\hline Complicaciones mayores & 6 & 0,8 \\
\hline Complicaciones & & \\
\hline intraoperatorias & 4 & 0,5 \\
\hline
\end{tabular}

gicas correspondieron a diez pacientes con sagrado vaginal profuso controlado en consultorio por vía vaginal, y una paciente con sangrado intracavitario corregido por laparoscopia.

De las lesiones vesicales una fue reconocida y corregida intraoperatoriamente y dos se manifestaron como fístulas vesico-vaginales que se corrigieron con manejo con sonda vesical por tres semanas. Se encontró una lesión ureteral $(0,1 \%)$ que se manifestó a los 14 días posoperatorios como una fístula vesico-ureteral derecha que requirió manejo urológico con reimplantación ureteral.
Las complicaciones clasificadas como "Otras" incluyen: dos pacientes con dolor pélvico severo, cinco con infección urinaria, dos con fiebre inexplicada, dos con vómito y diarrea posoperatorios, y una paciente con dolor pélvico severo a quien se le realizó laparotomía exploratoria sin encontrar alteraciones.

Todas las lesiones intraoperatorias correspondieron a lesiones de vías urinarias. No se presentaron lesiones intestinales, vasculares o defunciones en la actual serie de pacientes. En nuestro caso, la tasa de conversión a laparotomía fue del 2\% (14 pacientes) y todas se debieron a la patología intrínseca asociada a adherencias pélvicas severas o a tamaño excesivo del útero.

\section{DISCUSIÓN}

Nuestra cohorte de pacientes expuestas al manipulador mostró una tasa de complicaciones del 5,6\% (42 pacientes) de las cuales el 0,8\% (6 pacientes) fueron complicaciones mayores. Al igual que otros autores (19-21) no se consideró como complicación mayor la necesidad de convertir a laparotomía. Los datos mostrados son muy similares a los reportados por otros autores $(14,21-24)$ (tabla 3). La tasa de conversión a laparotomía también es similar a la reportada en otras partes $(14,24)$.

\begin{tabular}{|c|c|c|c|c|c|c|}
\hline Autor (año) $(\mathrm{n}=)$ & $\begin{array}{c}\text { Totales } \\
\text { (\%) }\end{array}$ & $\begin{array}{c}\text { Intra- } \\
\text { operatorias } \\
(\%)\end{array}$ & $\begin{array}{l}\text { Posope- } \\
\text { ratorias } \\
(\%)\end{array}$ & $\begin{array}{c}\text { Mayores } \\
\text { (\%) }\end{array}$ & $\begin{array}{c}\text { Menores } \\
\qquad(\%)\end{array}$ & $\begin{array}{l}\text { Uréter } † \\
\text { (\%) }\end{array}$ \\
\hline Corvalan (24), 2004 (144) & 9,8 & 5,6 & 4,2 & 5,6 & 4,2 & 0,7 \\
\hline Brummer (21), 2008 (13.942) & & 0,74 & & 1,0 & & 0,34 \\
\hline Garry (22), $2004(584)^{*}$ & 32,7 & 10,27 & 32,7 & 7,2 & 25,2 & 1 \\
\hline Navarro (23), 2005 (96) & 4,2 & 0 & 4,2 & 0 & 4,2 & 0 \\
\hline De los Ríos (24), 2009 (822) & 12,5 & 11,4 & 11,4 & 3,1 & 9,3 & 0,24 \\
\hline Argüello, 2012 (748) & 5,6 & 0,5 & 5,1 & 0,8 & 4,8 & 0,1 \\
\hline
\end{tabular}


El actual manipulador tiene la ventaja de lograr movilizaciones uterinas similares al de Rumi-Koh (16), “elevación” uterina y neumo-oclusión excelentes, y una mayor facilidad en su aplicación. Como desventajas de este movilizador están: la necesidad de dilatación cervical hasta el No. 10 de Hegar, el no brindar el eje de flexión uterina cerca del cérvix, lo que podría limitar su uso en pacientes muy obesas (16), el no tener un canal independiente para instilar líquidos intrauterinos, y el requerir un ayudante entrenado que se encargue de su movilización durante la cirugía.

El desempeño del M-R no fue adecuado cuando se intentó realizar histerectomía laparoscópica ampliada en los casos de lesiones de alto grado, ya que aunque la unión vagino-cervical se destacaba nítidamente, el punto de corte inferior para dejar un reborde vaginal adecuado era muy difícil de precisar.

Como limitaciones del estudio tenemos que no se dispuso de los datos de las complicaciones en el $12,4 \%$ de las pacientes, lo que puede llevar a una subestimación de las complicaciones asociadas al manipulador M-R.

\section{CONCLUSIÓN}

Con el uso del M-R se presentaron tasas de complicaciones similares a las reportadas por otros autores. Se requiere mayor investigación para definir cuál es el manipulador uterino ideal.

\section{REFERENCIAS}

1. Nieboer TE, Johnson N, Lethaby A, Tavender E, Curr E, Garry R, et al. Surgical approach to hysterectomy for benign gynaecological disease. Cochrane Database Syst Rev 2009;3:CD003677.

2. McPherson MA, Metcalfe A, Herbert M, Maresh A, Casbard J, Hargreaves, et al. Severe complications of hysterectomy: the value study. BJOG 2004;111:688-94.

3. Johnson N, Barlow D, Lethaby A, Tavender E, Curr L, Garry R. Methods of hysterectomy: systematic review and meta-analysis of randomised controlled trials. BMJ 2005;330:1478-85.
4. Munro MG, Parker WH. A classification system for laparoscopic hysterectomy. Obstet Gynecol 1993;82:624-9.

5. Reich H. Total laparoscopic hysterectomy: indications, techniques and outcomes. Curr Opin Obstet Gynecol 2007;19:337-44.

6. Leung SW, Chan CS, Lo SF, Pang CP, Pun TC, Yuen PM. Comparison of the different types of "laparoscopic total hysterectomy". J Minim Invasive Gynecol 2007;14:91-6.

7. Tanprasertkul C, Kulvanitchaiyanunt A. A modified technique to simplify TLH with new developed uterine manipulator; Anurach uterine manipulator (AUM). J Med Assoc Thai 2010;93 Suppl 7:154-9.

8. Mueller A, Oppelt P, Ackermann S, Binder H, Beckmann MW. The Hohl instrument for optimizing total laparoscopic hysterectomy procedures. J Minim Invasive Gynecol 2005;12:432-5.

9. Lee ET, Wong FW, Lim CE. A modified technique of LAVH with the Biswas Uterovaginal Elevator. J Minim Invasive Gynecol 2009;16:755-60.

10. McCartney A, Obermair A. Total lasparoscopic hysterectomy with a transvaginal tube. J Am Assoc Gynecol Laparosc 2004;11:79-82.

11. Koh C. A new technique and system for simplifying total laparoscopic hysterectomy. J Am Assoc Gynecol Laparosc 1998;5:187-92.

12. McCartney AJ, Johnson N. Using a vaginal tube to separate the uterus from the vagina during laparoscopic hysterectomy. Obstet Gynecol 1995;85:293-6.

13. Keriakos R, Zaklama M. The RUMI manipulator and Koh colpotomiser system for total laparoscopic hysterectomy. BJOG 2000;107:274-7.

14. Corvalán A, Roos A, Lattus J, Barrera V, Gallardo A, Flores J et al. Histerectomía laparoscópica: resultados en 3 años de desarrollo de la técnica utilizando el manipulador uterino de Clermont Ferrand. Rev Chil Obstet Ginecol 2004;69:446-50.

15. Perino A, Cucinella G, Venezia R, Castelli A, Cittadini E. Total laparoscopic hysterectomy: an assessment of the learning curve in a prospective randomized study. Hum Reprod 1999;14:2996-9. 
16. Mettler L, Nikam YA. A comparative survey of various uterine manipulators used in operative laparoscopy. Gynecol Surg 2006;3:239-43.

17. Argüello R, Hoyos E, Argüello R, Perdomo P. Histerectomía total por laparoscopia en 202 pacientes. Rev Colomb Obstet Ginecol 2003;54:258-64.

18. Argüello R, Hoyos E, Argüello R. Histerectomía total por laparoscopia: la nueva opción. Rev Colomb Obstet Ginecol 2001;52:359-65.

19. Chapron C, Fauconnier A, Goffinet F, Bréat G, Dubuisson JB. Laparoscopic surgery is not inherently dangerous for patients presenting with benign gynaecologic pathology. Results of a meta-analysis. Hum Reprod 2002;17:1334-42.

20. David-Montefiore E, Rouzier R, Chapron C, Darai E, Collegiale d'Obstétrique et Gynegologie de ParisIle de France. Surgical routes and complications of hysterectomy for benign disorders: a prospective observational study in French university hospitals. Hum Reprod 2007;22:260-5.
21. Brummer T, Sepäiä T, Härkki P. National learning curve for laparoscopic hysterectomy and trends in hysterectomy in Finland 2000-2005. Hum Reprod 2008;23:840-5.

22. Garry R, Fountain J, Mason S, Napp V, Brown J, Hawe J, et al. The eVALuate study: two parallel randomized trials, one comparing laparoscopic with abdominal hysterectomy, the other comparing laparoscopic with vaginal hysterectomy. BMJ 2004;328:129-36.

23. Navarro-Newball H. Histerectomía laparoscópica: sistematización del procedimiento y propuesta para programas de capacitación. Colomb Med 2005;36:115-9.

24. De los Ríos JF, Castañeda JD, Calle GA, Serna E, Vásquez R, Arango A et al. Histerectomía laparoscópica total en la unidad de endoscopia ginecológica de la Clínica del Prado, Medellín (Colombia) 2002-2008. Rev Colomb Obstet Ginecol 2009;60:320-7.

\section{Conflicto de intereses: ninguno declarado.}

\section{Financiación: para este trabajo no se recibieron ayudas financieras de entidad alguna. El manipu-} lador uterino descrito no se está produciendo comercialmente. 